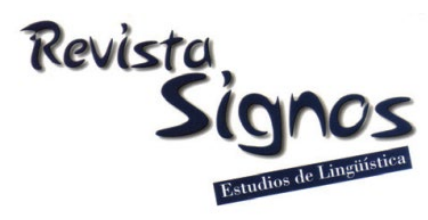

\title{
Los modales dinámicos en textos de historia en lengua inglesa (1700-1900)*
}

\author{
Dynamic modals in English history texts (1700-1900) \\ Francisco Alonso-Almeida \\ Universidad de Las Palmas de la Gran Canaria \\ ESPAÑA \\ francisco.alonso@ulpgc.es
}

Recibido: 02-I-2020 / Aceptado: 09-X-2020

DOI: $10.4067 /$ S0718-09342021000200529

\section{Resumen}

Los verbos modales han sido materia de análisis en la lingüística inglesa desde diferentes ópticas. Sin embargo, el estudio de los verbos modales en textos técnicos en la especialidad de historia no tiene gran trayectoria, a pesar de la información relevante que puede ofrecer un análisis de variantes diafásicas. Mi hipótesis de trabajo es que existe un uso significativo de perífrasis modales con valor dinámico, en el sentido que explica Palmer (2001). Mi interés en este trabajo se centra en comprobar el uso y la función que los modales dinámicos tienen en un corpus de textos técnicos en lengua inglesa en el registro de la historia en los siglos dieciocho y diecinueve. Este estudio, como se ha apuntado, pretende explorar el valor de la modalidad dinámica en el discurso científico, tal y como aparece en el Corpus of History English Texts (CHET), subcorpus que forma parte del Coruña Corpus of English Scientific Writing (1700-1900). La metodología que se emplea incluye el uso de herramientas propias de la lingüística de corpus para la localización y extracción de los datos. Además de los medios técnicos, se hace necesario una estimación de cada uno de los ejemplos rescatados de CHET para determinar con exactitud el valor dinámico de los verbos, así como su uso concreto en los contextos en los que aparecen. Los resultados de este análisis se reflejarán de manera cuantitativa, antes de presentar una visión cualitativa. Las conclusiones de este trabajo describen las formas usadas para la expresión de los significados dinámicos, así como la función que cumplen en los textos.

Palabras Clave: Modalidad, verbos auxiliares, disposición, potencialidad, inglés moderno. 


\begin{abstract}
English modal verbs have been the object of linguistic analysis from different perspectives. The study of modal verbs in technical texts in the domain of history, however, has not enjoyed a same interest, despite the relevant information an analysis of diaphasic variation may bring. My working hypothesis is that there is a significant use of modal verbs with a dynamic sense, as described in Palmer (2001). My interest in this work is to detect the use and function that dynamic modals have in a body of technical texts in English in history texts from the eighteenth and nineteenth centuries. This study, as already said, seeks to explore the value of dynamic modality in scientific writing that is in the texts compiled for the Corpus of History of Texts in English (CHET), subcorpus that is part of the Coruña Corpus of English Scientific Writing (1700900). The methodology used includes the use of corpus linguistics tools for the analysis and retrieval of data. In addition to the technical means, an inspection of each of the examples obtained from CHET is necessary to accurately determine the dynamic modal value of the verbs, as well as their specific use in the contexts in which they appear. The results of this analysis are given quantitatively along with presenting a qualitative interpretation of these results. The conclusions of this study includes an account of the modal forms with modal dynamic nuances, as well as their function in the texts.
\end{abstract}

Key Words: Modality, auxiliaries, disposition, potentiality, Modern English.

\title{
INTRODUCCIÓN
}

Los verbos modales han sido materia de análisis en la lingüística inglesa desde diferentes ópticas, suscitando el interés de los investigadores por la naturaleza tan diversa que han manifestado en su evolución. El estudio de los verbos modales en textos técnicos de historia, sin embargo, no tiene gran trayectoria, a pesar de la información relevante que puede ofrecer un análisis de las variantes diafásicas. Tanto es así, que mi hipótesis de partida incluye el predominio de modales con valor dinámico, en el sentido que explican Palmer (2001) y Vetter (2015), y que abarcaré de manera extensa en la sección 2. Ocurre, pues, que el uso expositivo de los textos técnicos favorece la presencia de modales que indiquen posibilidad inherente de personas, cosas y hechos para poder llevar a cabo una acción. Los textos técnicos describen numerosas situaciones que revelan acciones naturalmente potenciales según las capacidades de cada ser, cosa o hecho. En este sentido, mi interés se centra en comprobar el uso y la función que los modales dinámicos tienen en un corpus de textos técnicos en lengua inglesa en el registro de la historia en los siglos dieciocho y diecinueve.

Para muchos, los textos humanísticos y los históricos en particular suelen considerarse como menos técnicos que otros textos científicos que presentan un mayor grado de formalización, como pueden ser los textos sobre matemáticas, física o astronomía, por ejemplo. Lejos de ser así, los textos históricos representan un lugar idóneo para la argumentación científica, pues la génesis de conocimiento en esta disciplina requiere de un alto grado de evaluación de evidencias de naturaleza diversa, que den lugar a conclusiones lógicamente planteadas y reforzadas mediante estas 
evidencias. Como se apunta en Alonso-Almeida y Álvarez-Gil (2019: 151), los verbos modales constituyen un mecanismo lingüístico de gran fuerza argumentativa, pues permiten reflejar "the author's stance framing their subjective critical thinking".

Este estudio, como se ha dicho, pretende explorar el valor de la modalidad dinámica en el discurso científico, tal y como aparece en el Corpus of History English Texts (CHET), subcorpus que forma parte del Cornna Corpus of English Scientific Writing (1700-1900). La metodología que se emplea incluye el uso de herramientas propias de la lingüística de corpus para la localización y extracción de los datos. Además de los medios técnicos, se hace necesaria una estimación de cada uno de los ejemplos rescatados de CHET para determinar con exactitud el valor dinámico de los verbos, así como su uso concreto en los contextos en los que aparecen. Los resultados de este análisis se reflejarán de manera cuantitativa, antes de presentar una visión cualitativa de dichos resultados.

Este trabajo se estructura en cuatro secciones, además de esta introducción. En la sección 2, se consideran aspectos de importancia teórica para este estudio, tales como qué es la modalidad, la modalidad dinámica y los verbos modales ingleses. En la siguiente sección, se describe el corpus de textos históricos en lengua inglesa de los años 1700 a 1900, periodo de gran importancia para la especialización de los verbos modales en la historia de la lengua inglesa. Además, se exponen aspectos relativos a la metodología empleada para el análisis de los datos de estudio. A continuación, se presentan los resultados del estudio, además de las conclusiones, que se incluyen en la última sección.

\section{Modalidad y modalidad dinámica}

Palmer (1986: 2) define la modalidad como "something along the lines of Lyon's (1977: 452) 'opinion or attitude' of the speaker". Su descripción de modalidad incluye mecanismos gramaticales como los verbos modales o perifrásticos, el modo y ciertos clíticos, además de mecanismos léxicos, como los adverbios, que son capaces de indicar diferentes grados de perspectivización de la información. Frawley (1992: 385) indica que la "modality concerns the factual status of information; it signals the relative actuality, validity, or believability of the content of an expression". Narrog (2005) relaciona la modalidad con states of affairs, thus:

"Modality is a linguistic category referring to the factual status of a state of affairs. The expression of a state of affairs is modalized if it is marked for being undetermined with respect to its factual status, i.e. neither positively or negatively" (Narrog, 2005: 184). 
Lo expresado anteriormente refleja, sobre todo, el valor evaluativo y de perspectiva de la modalidad con respecto a la proposición modulada para expresar aspectos tales como probabilidad, posibilidad, obligación, permiso, necesidad, potencialidad, entre otros. Mediante determinados usos modales, se indica niveles de (inter)subjetividad del hablante con respecto a la proposición formulada. La función que cumplen puede, por ejemplo, relacionarse con estimaciones sobre la verdad de determinados hechos o con aseveraciones acerca de la realidad. En este contexto, se trataría de mitigar o de asegurar explícitamente la proposición, lo que, pragmáticamente, puede interpretarse como manifestaciones intencionadas de cortesía lingüística.

Son varias las clasificaciones de modalidad que se han planteado dependiendo de los enfoques epistemológicos que se sigan. Por lo general, la mayoría ofrece una visión binaria de los mecanismos modales. Una de estas propone la categoría epistémica y la deóntica. En cuanto a la primera, Nuyts (2001: xv) la define como "a speaker's evaluation of the likelihood of a state of affairs, as expressed in language ( e. g. "John is probably home")". La modalidad deóntica, por su parte, la explica de la siguiente manera: "Deontic modality is an evaluation of the moral acceptability, desirability or necessity of a state of affairs, i.e. it crucially involves notions such as 'allowance', 'permission' and 'obligation”' (Nuyts, 2001: 25), como en 'You cannot smoke here'.

Biber, Johansson, Leech, Conrad y Finegan (1999) utilizan también una clasificación similar, si bien usan una nomenclatura diferente. En este caso, se usan los términos 'modalidad intrínseca' y 'modalidad extrínseca', que se especifican así:

"Intrinsic modality refers to actions and events that humans (or other agents) directly control: meanings relating to permission, obligation, and volition (or intention). Extrinsic modality refers to the logical status of events or states, usually relating to assessments of likelihood: possibility, necessity, or prediction" (Biber et al., 1999: 485).

Sin embargo, hay una tercera categoría conocida como modalidad dinámica. Esta categoría la encuadra Palmer (2001) en lo que este autor denomina modalidad de evento (event modality) en inglés que forma, junto con la modalidad proposicional (propositional modality), su propuesta de modalidad. Esta última se relaciona con los usos modales epistémicos y se subdivide en la modalidad epistémica y evidencial - esto es, evidencia dada sobre el estatus de la proposición, como se apunta en Willet (1988). En el caso de la modalidad de evento, se refiere a las actitudes sobre acontecimientos del futuro y se subdivide, a su vez, en modalidad deóntica y modalidad dinámica. En la modalidad deóntica, se manifiestan sentidos de obligación y permiso, mientras que, en la modalidad dinámica, son sentidos de voluntad y habilidad por parte del orador o escritor, entre otros aspectos. Palmer (2001: 10) la define de la siguiente manera: "dynamic modality relates to ability or willingness, which comes from the individual concerned." 
Esta idea de habilidad y disposición se recoge también en Nuyts (2001), quien añade la idea de expresión de potencialidad y capacidad en el uso de este tipo de modalidad, como se aprecia en su definición:

"Dynamic modality involves an ascription of a capacity or a need co the subject-participant in the state of affairs, or of a situation-internal potential or necessity for him/her/it to do something (usually this involves animate entities, but it can also be extended to inanimate subject)" (Nuyts, 2001: 25).

En esta línea, y de acuerdo con Vetter (2015: 216), la modalidad dinámica, como se verá más adelante, está estrechamente relacionada con la "potentiality-based semantics" y, por lo tanto, a diferencia de otras categorías modales, "dynamic modality is a matter simply of how things really are, not how they ought to be or how we know them to be", lo que hace que, en ejemplos como Mary can speak, five languages, el uso de la partícula moduladora sea, hasta cierto punto, prescindible.

En este trabajo, me centro en este último tipo de modalidad, la dinámica, por ser una de las categorías menos abordadas en la literatura sobre modales en textos ingleses anteriores al siglo XX. Se estudian en particular los verbos modales con este significado. Denison (1993) describe los verbos modales según los criterios siguientes: (a) las formas modales no tienen formas finitas, (b) puede existir distinción temporal, aunque el uso de las formas en pasado puede indicar significados pragmáticos y no tiempo verbal, (c) no presentan desinencias verbales de persona y número, (d) admiten el uso de clíticos para expresar la negación, como, por ejemplo, can't, y pueden presentar formas reducidas, como $l l$ para shall y will, (e) estos verbos no se usan en imperativo, (f) se usa el infinitivo sin to con estos verbos, como en The boy can read well, (g) tienen una repercusión sobre el contenido proposicional modulando su significado, (h) puede darse la concurrencia de dos modales en determinados dialectos de la lengua inglesa, y finalmente (9) permiten el uso de la negación directo sin auxiliares, además de la inversión sujeto y verbo, la elisión de la proposición (función coda), y énfasis. Esta última descripción se conoce como propiedad NICE.

En inglés, los verbos modales centrales son 9, según Biber et al. (1999), a saber: can, could, may, might, shall, should, will, would, and must. Además, hay otros verbos modales llamados modales periféricos, marginales o semi-modales que incluyen las formas need (to), ought to, dare (to), used to. A pesar de que estos modales suelen ir seguidos por la estructura to-infinitivo, comparten algunas características con los modales centrales, tales como la negación directa, la inversión para formular preguntas, aunque dare y need pueden adoptar el auxiliar do/does. 


\section{Metodología, el Corpus of History English Texts y resultados del análisis}

El procedimiento que se ha usado para el análisis de los datos incluye metodología de lingüística de corpus. En este estudio, se ha empleado el Corpus of History English Texts (CHET) en una versión beta, cuya compilación se ha llevado a cabo en la Universidade A Coruña (véase Moskowich, Crespo, Puente-Castelo \& Monaco, 2019). Este corpus contiene ejemplos tomados de textos de historia escritos en el Reino Unido de Gran Bretaña y en lo que hoy se conoce como los Estados Unidos de América entre 1700 y 1900. Esto significa que nos encontramos ante la variante denominada inglés moderno tardío. Se trata, por lo tanto, de una compilación de alrededor de 400.000 palabras, la mitad corresponde a cada siglo, con representación desigual de autores y autoras. En el caso de los hombres, este subcorpus se compone de 322.849 palabras frente al subcorpus de textos escritos por mujeres, que contiene un total de 81.497 .

Este corpus, perteneciente al Coruna Corpus of English Scientific Writing (CC) dispone de su propia herramienta informática, Coruña Corpus Tool, ${ }^{1}$ que permite hacer búsqueda según criterios sociolingüísticos, como puede ser el sexo o la procedencia, y textuales de acuerdo con el género (tratado, libro de texto, etc.) que se trate (para una descripción de la herramienta véase Moskowich, Crespo, Lareo \& Camiña Rioboo, (2012) y Barsaglini-Castro y Valcarce (2020)). Su uso ha dado lugar a numerosos trabajos sobre género textual, evaluación del léxico, análisis de la perspectiva, efectos pragmáticos de uso de la lengua, entre otros (Moskowich, 2016, 2017a, 2017b; Crespo, 2017; Álvarez-Gil, 2018; Moskowich \& Crespo, 2018, 2019; Alonso-Almeida \& Álvarez-Gil, 2019).

En CHET, se ha buscado de manera computarizada concordancias que contengan los siguientes verbos modales centrales: can, could, may, might, shall, should, will, would y must. Una vez obtenidos los ejemplos, se ha procedido a un análisis manual de evaluación del contexto para determinar el significado exacto de cada modal, para poder así aislar aquellos que pueden ser categorizados como modales dinámicos. Con ello, se pretende determinar el uso específico de estos verbos en la prosa científicotécnica de la época y mostrar los usos de este tipo de modalidad en este registro científico.

\section{Resultados del análisis}

En total se han encontrado 3501 verbos centrales, repartidos entre las formas que se aprecian en el siguiente gráfico: 


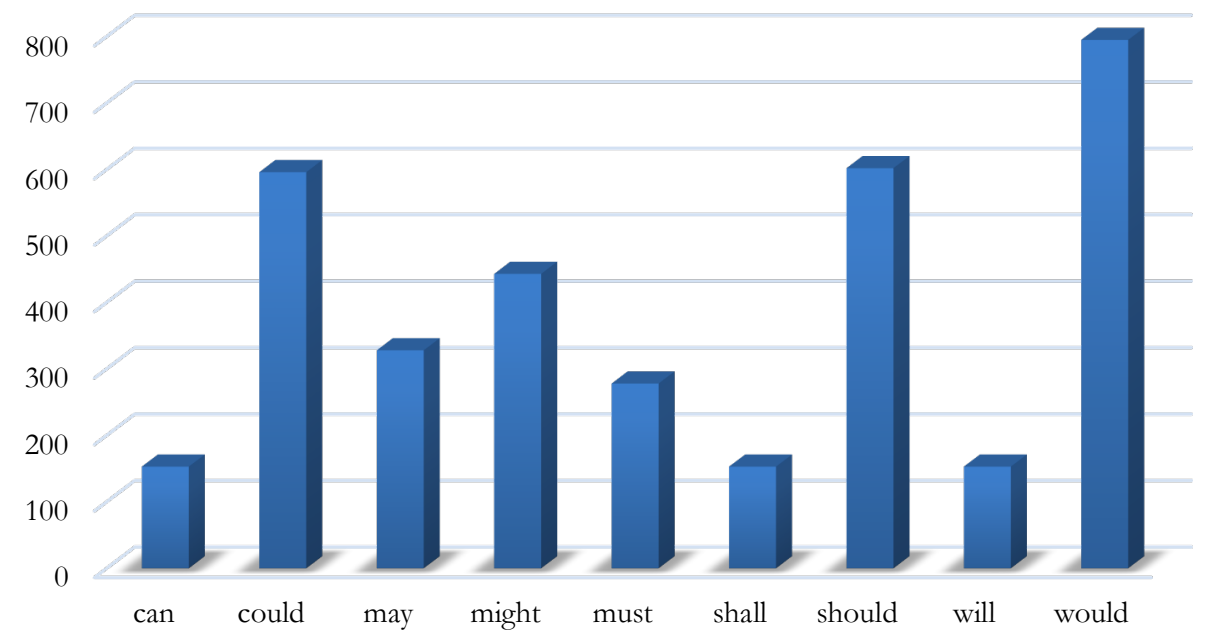

Gráfico 1. Verbos modales en CHET.

Estos verbos se distribuyen a su vez en las tres modalidades descritas en la sección 1, así: 43\% de los casos son epistémicos, 36\% de ellos son deónticos y 21\% son dinámicos. Es evidente, pues, que es la modalidad dinámica la que menor incidencia presenta, aunque su presencia es importante. La modalidad epistémica ocupa un lugar destacado, lo que es comprensible, pues este tipo permite establecer generalizaciones y aseveraciones de manera atenuada con el fin de garantizar unos principios básicos de modestia académica que eviten la imposición al autor de lo que se dice. En esta línea, la mitigación del contenido proposicional mediante estos modales persigue rebajar las expectativas sobre la realidad descrita situándola en el terreno de lo probable y, por lo tanto, también se rebaja la responsabilidad de los autores y autoras por la información que se presenta. Esto parece especialmente útil en los estudios de historia donde parte de la información se desarrolla mediante la interpretación de los hechos a través de testimonios, objetos y documentos sin que sea siempre posible la verificación de estos hechos. Esto ocurre con los verbos epistémicos de necesidad que presentan inferencias.

Los verbos deónticos, segundos en frecuencia, se usan para indicar diferentes grados de obligación, necesidad, imposición interna o externa y la concesión de permiso. Estos valores de los verbos deónticos rara vez se perciben con una fuerza de obligación por parte de los autores acerca de lo que informan. Casi siempre se refieren a causas que generan necesariamente un hecho o, incluso, a las acciones de leyes naturales o divinas que inciden en la libertad de acción para la realización de la actividad que se describe. Otro de los significados asociados con la modalidad deóntica en estos textos es la recomendación de que se realice una acción determinada. En cuanto a los verbos dinámicos, con menor representación, se mostrarán sus usos en la sección 3. 
En ocasiones, la diferencia entre los verbos modales con valor dinámico y con valor epistémico no siempre es fácil de identificar, pues la noción de lo que es potencialmente posible puede hallarse bajo la posibilidad o la probabilidad de que una acción se realice o no, como en el ejemplo tomado de CHET we might have the advantage of his assistance in our endeavour to ascertain how far the various monuments still remaining could be identified with those anciently described (Petrie, 1839), en el que se parte de unas características propias de un objeto para establecer una hipótesis. Todos estos casos han sido categorizados como modales epistémicos.

También se han descartado aquellos casos que den lugar a significado inferencial que, de acuerdo con Van der Auwera y Plungian (1998), pertenecen al espacio semántico de la necesidad epistémica, incluso si el contexto indica la consideración de las cualidades o de la disposición de las personas u objetos en la realización de una acción determinada. En el ejemplo de CHET, But no Peace could have lasted under such bumiliations as Austria, Prussia, Spain, Italy, and the Low Countries had been forced to undergo (Burrows, 1895), la propia noción del término paz y su significación entre países no es compatible con las humillaciones, por lo que la deducción es posible y justificada. Este tipo de mecanismo modal es útil en los textos de historia para formular información de manera circunstancial, que no siempre puede ser probada. Todos estos casos se han clasificado como modales epistémicos de necesidad y no se han considerado en este estudio.

\subsection{Los modales dinámicos en CHET}

Se han identificado 675 verbos modales con valor dinámico en los textos usados de CHET y se distribuyen en las formas can, could, may y would, de la manera que se indica en el gráfico que sigue de acuerdo con el sexo del autor/a:

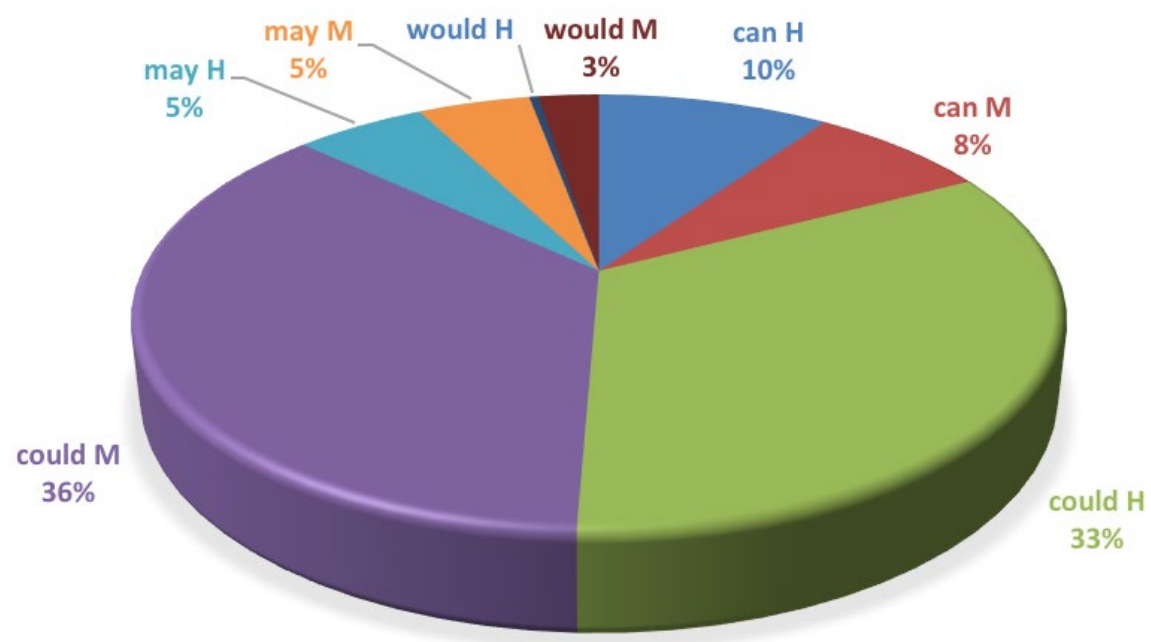

Gráfico 2. Los verbos modales dinámicos en CHET H=hombre; $\mathrm{M}=$ mujer). 
La forma modal más frecuente con valor dinámico es could, con un $69 \%$ de todos los casos analizados, con más ocurrencias en el caso de los textos escritos por mujeres. El verbo would, por el contrario, solo aparece en un 3,1\% con este significado con la casi totalidad de los ejemplos en el subcorpus de textos escritos por mujeres. El resto de formas en este grupo, can y may, aparecen con un 18\% y un 10\%, respectivamente. Estos datos son muy interesantes, pues Gisborne y Holmes (2007: 45) apuntan que "CAN is the only modal which clearly retains a dynamic sense". Según los autores, Palmer (2003) identifica valores modales dinámicos también en la forma will. Sin embargo, will no aparece en CHET con este valor y can no es el único verbo modal en los textos que se han examinado en este estudio con un uso dinámico. Huddleston y Pullum (2002) explican que, a pesar de que can parece ser la forma dinámica con menor número de restricciones, existen otras como may, que puede aparecer como sustituto de can en registros formales, y como must, que expresa un significado de necesidad dinámica. En esta sección, se describen los usos dinámicos de can, may, would y could en CHET.

\subsection{Valores dinámicos de los modales can, may, would, may, would y could en CHET}

Los valores dinámicos representados en CHET se relacionan con aspectos de capacidad y disposición que he agrupado en tres usos según su significado, a saber: (a) capacidad, (b) ausencia de habilidad para llevar a cabo una acción, y (c) estar en posición de/condición de poder realizar una acción, cuya distribución se ofrece de manera global por uso y sexo en el Gráfico 3 y por sexo y modal en el Gráfico 4, con datos normalizados:

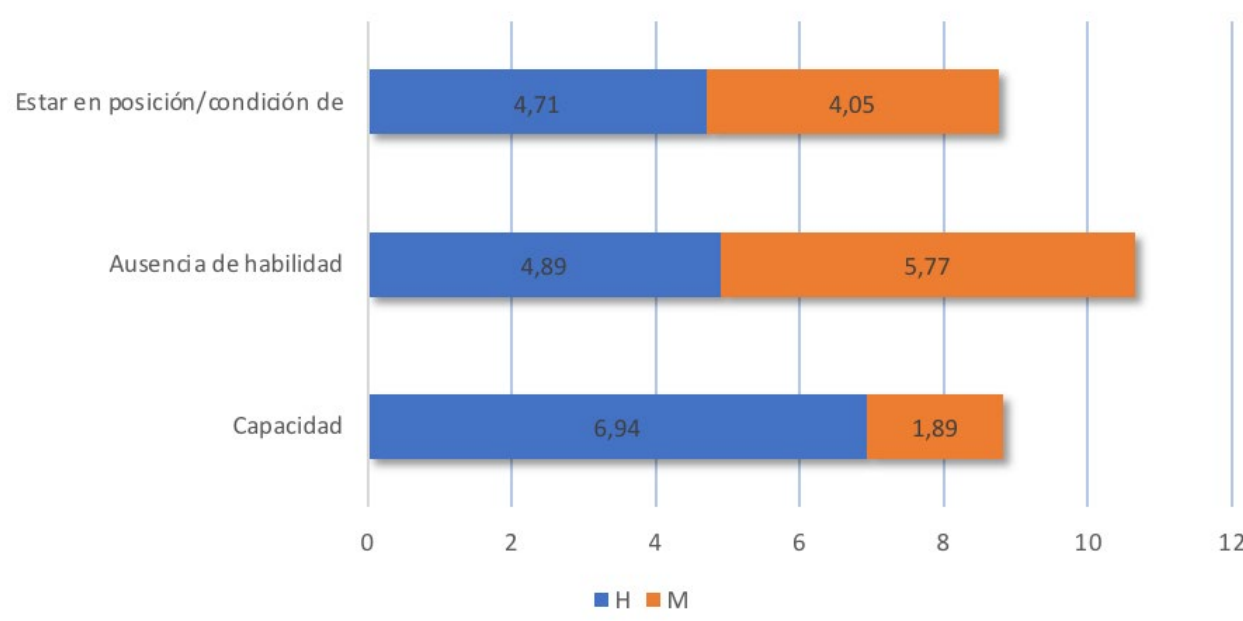

Gráfico 3. Uso global de los modales dinámicos en CHET H=hombre; M=mujer) N/10.ooo palabras. 


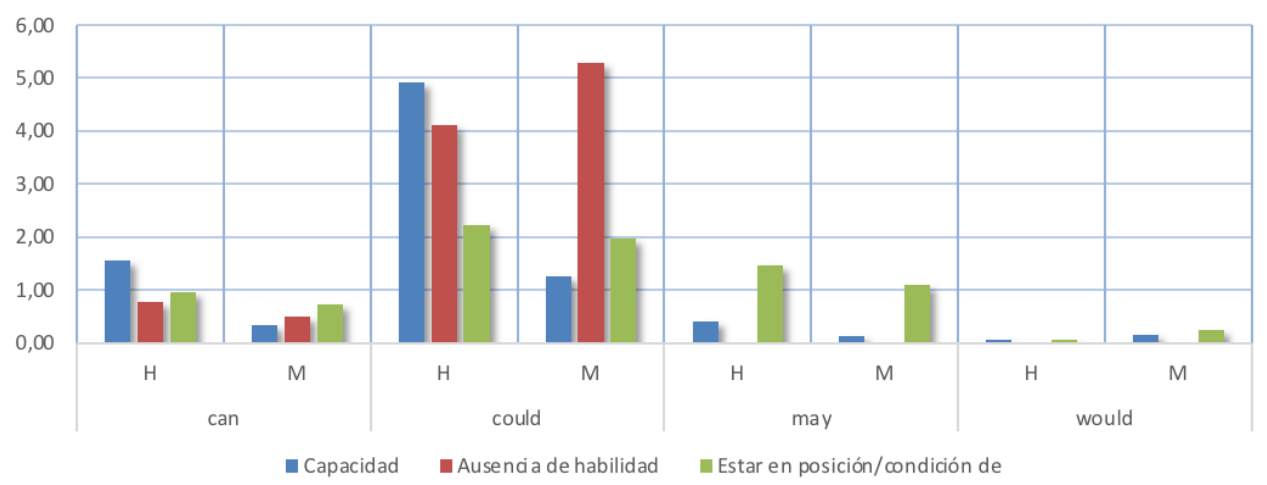

Gráfico 4. Distribución de los modales dinámicos por uso en CHET H=hombre; $\mathrm{M}=$ mujer) N/1000o palabras.

Los datos reflejados en el Gráfico 3 revelan que el uso dinámico más frecuente en CHET es la ausencia de habilidad y, en este sentido, se muestra casi la misma proporción en su empleo independientemente del sexo de sus autores. Como se ve en el Gráfico 4, tanto las autoras como los autores tienen una preferencia evidente por could para manifestar este uso, seguido muy de lejos por can. El siguiente valor en frecuencia es el significado de capacidad, que aparece muy destacado en el subcorpus de textos escritos por hombres y cuya realización se indica preferentemente mediante could, seguido en menor medida por can. Esta tendencia es la misma en el subcorpus de mujeres, si bien este uso es menos usual. El uso para significar estar en posición también es más común en los textos escritos por hombres y los verbos modales usados para este fin son could, con igual frecuencia en los dos subcorpus, seguido de may y can, más usados en el subcorpus de hombres que en el de mujeres. Destaca que no se usa el verbo may para indicar ausencia de habilidad.

\subsection{Descripción de forma de los usos dinámicos en CHET}

En esta sección se ejemplifican los valores dinámicos anteriormente mencionados por orden de frecuencia para mostrar su realización en los siglos dieciocho y diecinueve. Posteriormente, se describe las funciones que tienen estas estructuras en el discurso académico en estos siglos.

\subsubsection{Ausencia de habilidad o imposibilidad}

Las formas modales que se emplean para expresar la ausencia de habilidad o la imposibilidad para llevar a cabo una acción son could y can, por orden de preferencia, y son las mujeres quienes emplean could con mayor frecuencia. Los siguientes ejemplos muestran este uso:

1. And in thefe refpects, fuch was the Condition of the Place, that our Ambalfadors, in their Letters to Cardinal Wolfey, faid, they could not tell how the Pope could be faid to be at Liberty, being there, where Hunger, Scarcity, ill- 
favoured Lodging, ill Air, and many other Incommodities, kept him and all his as ftreightly, as he was ever Captive in Caftle Angel (Strype, 1721).

2. Knowing besides, that the Army he was to command, tho' formidable in Number, was only a Multitude of undisciplined Peasants, with whom he could never undertake any daring Action to come off with Honour (Bancks, 1740).

3. Cyrus replied "that he was bound by the king's orders, and that it was settled by treaty how much money should be given to the Lacedæmonians to assist them in keeping up their fleet, so that it was not in his power to grant Lysander's request." Lysander, of course, could say no more at that moment (Sewell, 1857).

Todos los ejemplos indicados aquí coinciden en la presencia de adverbios de negación, o expresiones que indican negación, como not, never y no more que polarizan el significado de habilidad o posibilidad de llevar a cabo una acción, ya sea en condición permanente, como en los ejemplos escritos por hombres en (1) y (2), o circunstancial como en la muestra (3) de la autora Sewell. En este último, la imposibilidad es externa y no depende de una disposición interna o de una habilidad del actor. En el caso del ejemplo (1), se trata de una imposibilidad interna por la que el hablante admite no ser capaz de comprender lo que dice el Papa dado el contexto adverso del que se habla.

Igualmente, can se usa para expresar falta de habilidad o posibilidad de realizar una acción por cuestiones internas o externas al sujeto de dicha acción, como se observa en los siguientes ejemplos; el primero de ellos escrito por un hombre y el segundo por una mujer:

1. Indeed, King James kept his Intention as fecret as he could; and no body but his Queen and Father Petres were inform'd of it. When the latter propos'd to him, to let Tyrconnel know what he intended to do for him: The King reply'd, 'Tis not Time yet; and He can't keep a Secret (Oldmixon, 1716).

2. And now if the church will not hold up themselves under God, I can do no more (Aikin, 1833).

En el primer caso, se usa la forma contraída de can en negativo para expresar la ausencia de disposición interna del hablante y, consecuentemente, la imposibilidad de poder guardar un secreto, como se formula en el ejemplo. En el caso siguiente, también aparece una estructura negativa, esto es no more, que indica la inviabilidad de que el sujeto pueda hacer algo más, al menos circunstancialmente de acuerdo con el condicionante que se expresa en la oración de if. 


\subsubsection{Capacidad}

En consonancia con el uso anterior, en este caso, se trata de expresar capacidad, incluido significados de disposición, para llevar a cabo una acción. Este valor se manifiesta en el uso de los modales could, can, may y would, en este orden de frecuencia. La forma could es la más común de ellas, seguida de can, mientras que may y could aparecen de manera esporádica. Los siguientes ejemplos ilustran este significado:

1. This Author relates, that the Duke had much difpleas'd the King by his Tharp and undutiful Speeches, tho' His Majefty ftill endeavour'd to oblige him, by all the Favours He could heap upon him; fo that he proceeded not only to fecret Caballs, but open Confpiracies (Tyrrell, 1704).

2. But as it does not appear that any wise or good end could be answered by this dream, as his wife was dead before he could possibly come to her assistance, ought it not to be ascribed to those fancies of the brain of which no rational account has been yet given (Cornish, 1780).

3. Eric's youth was his greateft recommendation; and the preferred him, becaufe it would be many years before he could become a dangerous competitor (Scott, 1762) --female.

En todos los ejemplos anteriores de could, se refieren a la capacidad de los sujetos para la realización del contenido proposicional, a saber heap upon him en (6), possibly come to her assistance en (7), y become a dangerous competitor en (8). En todos los casos, se trata de capacidades externas temporales sin relación con las cualidades o características innatas de los sujetos de las acciones descritas. En el caso de (7), se aprecia el empleo del mecanismo epistémico possibly, que afecta al contenido proposicional, pero no al valor dinámico de could. La idea de disposición con can y may se observa también en los ejemplos siguientes escritos por mujeres:

1. The common Men can go through great Hardhips; and will live in Places, and on fuch mean Food, that would kill our Natives (Justice, 1739).

2. As to the Ruffia Climate, it is extraordinary cold, as you may imagine: For I faw Two and Thirty Thoufand Men exercifed upon the Ice (Justice, 1739).

Mientras que can en (9) se refiere a una disposición que es posible de acuerdo con las características propias de los common men, que los hacen adecuados para la acción descrita, el uso de may en (10) indica que el hablante tiene la capacidad de imaginarse el frío extraordinario que hace en Rusia por información general que se tiene de este país y su climatología. A esto debe añadirse la capacidad interna de una persona para establecer relaciones mentales lógicas, por lo que esta disposición intrínseca juntamente con la información aprendida acerca de Rusia permite la realización del contenido de la proposición. 
Este uso dinámico que se basa en la capacidad para el desarrollo de información histórica es más frecuente en los textos escritos por hombres que en aquellos que han sido escritos por mujeres. Esto se lleva a cabo mediante un uso mayoritario de could con este significado en los hombres, pero se usa principalmente para indicar ausencia de habilidad o imposibilidad en las mujeres. La relación obvia que existe entre estos dos grupos hace que esta variación no sea realmente significativa.

\subsubsection{Estar en posición de/condición de}

El valor dinámico al que se hace referencia en esta sección indica aspecto de posibilidad de llevar a cabo una acción en términos de capacidad teniendo en cuenta la existencia de ciertos aspectos condicionantes que son "suitable for its exercise" (Vetter, 2015: 215), como se puede observar en los ejemplos siguientes:

1. Nero is thought to have died on the tenth of June, to which if a year and twenty two days be added, it will bring the beginning of Vefpafian's reign near the firft of July. So that I think upon the whole we may reckon Vefpafian's reign from the firft of July (Horsley, 1732).

2. It was in this extremity that [Madlle]. Mance agreed to place in the hands of de Maisonneuve 22,000 francs received from Madame de Bullion for the Hospital. By these means he could proceed to France and obtain additional force (Kingsford, 1887).

3. Even in Attica itself they had obtained a fixed settlement; for they possessed a fortress, called Decelea, so near to Athens that they could almost see from it the great public processions of the Athenians on their festival days (Sewell, 1857).

En estos ejemplos, se observa que el valor dinámico de may and could indica que la proposición $(P)$ es posible en (13) según las premisas expuestas, por lo que we may reckon $P$ se presenta como una causa introducida por so that. Igualmente, en (11) y (12) redactado por un escritor y una escritora, respectivamente, el modal could indica que existe una disposición para la realización de $P$ en estos casos en los que se refieren a eventos logrados en el pasado y esta se logra a través de la existencia de un contexto habilitante para que se produzcan. De ahí, la presencia de by these means en (12) y de so $A D J$ that que revelan el proceso argumentativo en el que los historiadores formulan su información. En esta misma línea, el modal may en los siguientes textos del historiador Gibbon (1788) y la historiadora Cooke (1893), en (14) y (15) respectivamente, presenta un poderoso valor argumentativo, pues viene determinado por las evidencias expuestas, como se puede observar incluido la presencia del mecanismo indicador de causalidad therefore: 
1. But the wealth of the province, and the trult of the revenue, were founded on the fair and plentiful produce of trade and manufactures: and fome fymptoms of liberal policy may be traced in a law which exempts from all perfonal taxes the mariners of Peloponefus, and the workmen in parchment and purple. This denomination may be fairly applied or extended to the manufactures of linen, woollen, and more efpecially of filk: the two former of which had flourifhed in Greece fince the days of Homer; and the laft was introduced perhaps as early as the reign of Juftinian (Gibbon, 1788).

2. It is confirmed, moreover, by two manuscripts in the Cottonian collection belonging to the thirteenth century. We may therefore conclude that some formality was lacking in the earlier year which would prevent that date, namely, 24 [Nov]. 1128, from being regarded as the true birthday of the monastery (Cooke, 1893).

El verbo modal can también presenta una función clara en los procesos deductivos de elaboración de información en los textos de CHET, como se aprecia en los ejemplos de Chapman (1750), Hardiman (1820) y la autora Sewell (1857):

1. The addition, which was made to the fenate, in confequence of the peace with the Sabines, and their incorporation with the Roman people, is not mentioned with any circumftances, that can lead us to think, any alteration was then made in the election of fenators: nay, on the contrary, we are exprefsly told, that the number of patricians being doubled, by chuling as many Sabines into that order, as there were Romans of it before, the people afterwards, in an affembly held by curias, elected a hundred of them into the fenate, which, by this means, was increafed to twice the number, of which it confifted, as its firft inftitution (Chapman, 1750).

2. Considering, therefore, that these facts are no where respectably controverted, but stand on as firm a foundation of historical authority as, under all the circumstances, can reasonably be expected at the present day (Hardiman, 1820).

3. The battle which at last ended the long Peloponnesian war, is called the battle of Ægos Potami. It was fought near Sestos, in Asia Minor; but it can scarcely be called a regular engagement, and it would probably never have taken place if the Athenians had not been strangely negligent (Sewell, 1857).

En estos casos, can contribuye a la línea argumentativa de estos textos, pues refleja en ellos que las proposiciones que siguen a este verbo modal son posible como hechos circunstanciales condicionados por la información previa que se dispone. De estos ejemplos, es especialmente evidente la muestra en (16), que presenta detalles 
contextuales y argumentativos muy destacados, que incluye evidenciales intersubjetivos como we are expressly told.

\section{Discusión}

Los datos analizados revelan que no existe una variación significativa en cuanto al uso de los modales con significado dinámico según la variable de sexo. La modalidad dinámica en CHET no parece estar vinculada exclusivamente a las características propias de los objetos, los eventos o las personas. Existe un uso circunstancial determinado por el contexto en el que se incluyen los verbos modales en cuestión y que revelan su empleo en el entramado argumentativo de los textos técnicos en la especialidad de historia en los siglos dieciocho y diecinueve. La pregunta que se plantea es, además de este uso, qué otra función puede cumplir estos modales.

Una función que parece evidente en el empleo de la modalidad dinámica es su valor para indicar factualidad basados en un determinado tipo de posibilidad que se deriva de la disposición de entes, por ejemplo, para abarcar una actividad específica, como se observa en los casos, a continuación:

1. BY this, we may fee what Faith and Credit is to be given to Englifh Hiftorians, when any palfage ferves to exalt and aggrandize their Prince, and leffen others (Anderson, 1705).

2. ....in the enfuing elections of fenators, I think, we may very fairly prefume, that they continued to exercife the fame right, in every fubfequent inftance, which we have already lhewn them to have done, in the firft (Chapman, 1750).

En estos dos ejemplos, los autores usan la primera persona del plural de manera inclusiva, por lo que incide en el valor dinámico de may, que se usa para indicar la disposición de los sujetos para llevar a cabo los procesos mentales see 'entender' y presume 'suponer' que demandan de las habilidades internas de los participantes en su realización. En el caso de la muestra en (20), además de este uso incluyente del pronombre we, se ofrece la perspectiva del autor mediante el uso del mecanismo epistémico I think, que se aporta de manera parentética precediendo we may $P$. Este uso puede tener un efecto mitigante del contenido proposicional que se aporta para evitar la imposición de su criterio. Por otra parte, el uso del evidencial fairly precedido por el intensificador very se presenta como un mecanismo con claro valor deíctico, por lo que, en su conjunto, podría tener un efecto descortés. Esto no significa que exista un ánimo de descortesía por parte del escritor, pues:

"Impoliteness is very much in the eye of the beholder, that is, the mind's eye. It depends on how you perceive what is said and done and how that relates to the situation," como apunta Culpeper (2011: 22). 
Dicho esto, y lejos de buscar una significación especial al uso de may, que no sea una indicación del papel que juegan los sujetos en los procesos cognitivos descritos en los ejemplos, el uso de esta forma modal guarda relación con tratamientos formales asociados a la cortesía lingüística (Collins, 2009), al menos, cuando este modal se usa en sentido epistémico. Podría, por lo tanto, entenderse este efecto pragmático de atenuación como una extensión cuando may tiene un significado dinámico.

Esta extensión del significado de cortesía no es tan evidente cuando se emplean otras formas modales dinámicas como, por ejemplo, el verbo can, en los siguientes ejemplos, aunque sí se percibe con una fuerza similar a la de may en el ejemplo con could:

1. THIS feems to be the whole of what we can know of the Roman tranfactions in Britain during the reign of Marcus Antoninus (Horsley, 1732).

2. The joy of the British at the cessation of the war led the upper classes to disregard all risks in order to gratify the intense passion for foreign travel which had for centuries been characteristic of the aristocracy. Not that any one could be fairly supposed to guess that if the war broke out again Napoleon would detain every English man and woman within his dominions, a proceeding worthy of an Oriental despot. That was only the final act of aggression (Burrows, 1895).

Estos ejemplos indican que la modulación del contenido proposicional mediante modalidad dinámica puede tener una intención de mitigación lingǘstica, pero este no aparenta ser su función principal. Por otra parte, el uso de la modalidad dinámica en nuestros textos parece poner en evidencia el papel que juegan los entes y sus peculiaridades, en términos de disposición y habilidad, en el desarrollo argumentativo de los textos. En algunos casos, como se ha visto, los verbos modales dinámicos permiten identificar el papel del sujeto y su implicación en la ejecución de una acción determinada.

\section{CONCLUSIÓN}

En este trabajo, se han presentado ejemplos del uso de modales dinámicos en textos de los siglos dieciocho y diecinueve en la especialidad de historia escritos por mujeres y hombres. Los datos que se han obtenido mediante el análisis computarizado de los textos revelan que solo hay cierta variación en el uso de la modalidad dinámica en el grupo que se refiere a la capacidad de realizar la acción descrita en la proposición. Sin embargo, como se ha apuntado, esta diferencia no es significativa, si consideramos que el dominio semántico está relacionado con el grupo denominado ausencia de habilidad, que se usa más en el caso de los textos escritos por mujeres. 
Una de las conclusiones más relevantes de este trabajo es el uso de la modalidad dinámica en el entramado argumentativo del texto técnico sobre historia. Este potencial viene determinado por el contexto que provee evidencias para determinar la información modulada y que, en ocasiones, reflejan la implicación de los autores o de los sujetos de las acciones que se manifiestan en la elaboración de la información. En este sentido, en todos los casos descritos en este trabajo, los verbos dinámicos estaban acompañados de otras estrategias argumentativas de causalidad o evidenciales, lo que redundaba en la idea de conclusión lógica de los argumentos aportados. Este método de presentar la información es muy destacado en esta disciplina donde, con frecuencia, se carece de evidencias documentales que puedan ilustrar todos los momentos históricos, por lo que se ha de recurrir a la deducción o la hipótesis. La elaboración de hipótesis en estos textos también se desarrolla a través de la descripción de las capacidades o disposiciones de entes y objetos, aunque estas puedan ser circunstanciales, tal vez por cuestiones externas y no internas a los hablantes. Entre las formas más usadas para significar cortesía están may y could, probablemente como extensión de sus equivalentes epistémicos.

La función pragmática de los modales dinámicos es principalmente manifestar cortesía mediante la atenuación del contenido proposicional para evitar imponer el punto de vista de los hablantes. La modalidad dinámica, que se refiere a una posibilidad basada en la disposición y el potencial para realizar una acción, se basa en esta característica para conseguir este efecto mitigante, pues se presenta a menudo la información aportada como una perspectiva y no como un hecho constatado. En esta línea, se entiende, y es la idea que he tratado de defender en este trabajo, que la modalidad dinámica en el corpus de trabajo persigue con frecuencia mostrar la identificación del papel del hablante en la elaboración de la información, ya sea por referencia a características internas o externas de los participantes.

\section{REFERENCIAS BIBLIOGRÁFICAS}

Alonso-Almeida, F. \& Álvarez-Gil, F. J. (2019). Modal categories in CHET. En I. Moskowich, B. Crespo, L. Puente-Castelo \& L. Maria Monaco (Eds.), Writing History in Late Modern English: Explorations of the Coruña Corpus (pp. 150-165). Ámsterdam, Nueva York: John Benjamins.

Álvarez-Gil, F. J. (2018). Adverbs ending in -ly in Late Modern English. Evidence from the Coruña Corpus of History English Texts. Valencia: Editorial Universitat Politècnica de València.

Barsaglini-Castro, A. \& Valcarce, D. (2020). The Coruña corpus tool: Ten years on. Procesamiento del lenguaje natural, 64, 13-19.

Biber, D., Johansson, S., Leech, G., Conrad, S. \& Finegan, E. (1999). Longman grammar of spoken and written English. Londres: Longman. 
Burrows, M. (1895). The History of Foreign Policy. Edimburgo y Londres: Blackwood \& Sons.

Collins, P. (2009). Modals and quasi-modals in English. Ámsterdam, Nueva York: Rodopi.

Crespo, B. (2017). Creating an identity of persuasion in history texts. En F. AlonsoAlmeida (Ed.), Stancetaking in Late Modern English Scientific Writing. Evidence from the Coruña Corpus. Colección Scientia [Applied Linguistics] (pp. 41-55). Valencia: Editorial Universitat Politècnica de València.

Culpeper, J. (2011). Impoliteness: Using language to cause offence. Cambridge: Cambridge University Press.

Denison, D. (1993). English historical syntax: Verbal constructions (Longman Linguistics Library). Londres, Nueva York: Longman.

Frawley, W. (1992). Linguistic semantics. Hillsdale: Erlbaum.

Gisborne, N. \& Holmes, J. (2007). A history of English evidential verbs of appearance. English Language and Linguistics, 11(1), 1-29. https:/ /doi.org/10.1017/S1360674306002097

Huddleston, R. \& Pullum, G. K. (2002). The Cambridge grammar of the English language. Cambridge: Cambridge University Press.

Lyons, J. (1977). Semantics. Cambridge: Cambridge University Press.

Moskowich, I. (2016). Lexical richness in modern women writers: Evidence from the Corpus of History English Text. Revista Canaria de Estudios Ingleses, 72, 111 128.

Moskowich, I. (2017a). Genre and change in the Corpus of History English Texts. NJES Nordic Journal of English Studies, 16(3), 84-106. https://doi.org/10.35360/njes.413

Moskowich, I. (2017b). Pronouns as stance markers in the Coruña Corpus: An analysis of the CETA, CEPhiT and CHET. En F. Alonso-Almeida (Ed.), Stancetaking in Late Modern English Scientific Writing. Evidence from the Coruña Corpus. Colección Scientia [Applied Linguistics] (pp. 73-91). Valencia: Editorial Universitat Politècnica de València.

Moskowich, I. \& Crespo, B. (2018). Categories and genres in CHET and CECHeT. En A. Moreno Ortiz \& C. Pérez Hernández (Eds.), International Conference on Corpus Linguistics. EPiC Series in Language and Linguistics, 1, 308-294. https://doi.org/10.29007/kvjx 
Moskowich, I., \& Crespo, B. (2019). "Arguments That Could Possibly Be Urged": Modal Verbs and Tentativeness in the Coruña Corpus. Languages, 4(57), 1-12. https:/ / doi.org/10.3390/languages4030057

Moskowich, I., Crespo, B., Lareo, I. \& Camiña Rioboo, G. (2012). Astronomy 'playne and simple.' Ámsterdam: John Benjamins. https://doi.org/10.1075/z.173

Moskowich, I., Crespo, B., Puente-Castelo, L. \& Monaco, L. M. (2019). Writing history in late modern English. Ámsterdam: John Benjamins. https://doi.org/10.1075/z.225

Narrog, H. (2005). On defining modality again. Language Sciences, 27(2), 165-192. https:/ / doi.org/10.1016/j.langsci.2003.11.007

Nuyts, J. (2001). Epistemic modality, language, and conceptualization: A cognitive-pragmatic perspective. Ámsterdam, Nueva York: John Benjamins.

Palmer, F. R. (1986). Mood and modality. Cambridge: Cambridge University Press.

Palmer, F. R. (2001). Mood and Modality. Cambridge: Cambridge University Press. https://doi.org/10.1017/CBO9781139167178

Palmer, F. (2003). Modality in English: Theoretical, descriptive and typological issues. En R. Facchinetti, M. Krug \& F. R. Palmer (Eds.), Modality in contemporary English (pp. 1-17). Berlin: Mouton de Gruyter.

Petrie, G. (1839). On the History and Antiquities of Tara Hill. Transactions of the Royal Irish Academy, 18, 25-232.

Van der Auwera, J. \& Plungian, V. A. (1998). Modality's semantic map. Linguistic Typology, 2(1), 79-124. https://doi.org/10.1515/lity.1998.2.1.79

Vetter, B. (2015). Potentiality. From dispositions to modality. Oxford: Oxford University Press.

Willett, T. (1988). A cross-linguistic survey of the grammaticization of evidentiality. Studies in Language, 12(1), 51-97. https:/ /doi.org/10.1075/sl.12.1.04wil

\section{* AGRADECIMIENTOS}

Esta investigación se ha llevado a cabo gracias a los proyectos FFI2016-75599-P y FFI2016-77941-P concedidos por el Ministerio de Economía, Industria y Competitividad (MINECO). 


\section{NOTA}

${ }^{1}$ Herramienta disponible en. https://ruc.udc.es/dspace/handle/2183/21850. 